\title{
Pair Spectrometer Hodoscope for Hall D at Jefferson Lab
}

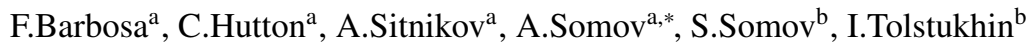 \\ ${ }^{a}$ Thomas Jefferson National Accelerator Facility, Newport News, VA 23606, USA \\ ${ }^{b}$ National Research Nuclear University MEPhI, Moscow, Russia
}

\begin{abstract}
We present the design of the pair spectrometer hodoscope fabricated at Jefferson Lab and installed in the experimental Hall D. The hodoscope consists of thin scintillator tiles; the light from each tile is collected using wave-length shifting fibers and detected using a Hamamatsu silicon photomultiplier. Light collection was measured using relativistic electrons produced in the tagger area of the experimental Hall B.

Keywords: Scintillator detector, Pair spectrometer, Silicon photomultiplier
\end{abstract}

\section{Introduction}

The new detector GlueX has been constructed in the experimental Hall D at Thomas Jefferson National Accelerator Facility. The main physics goal of the GlueX experiment [1] is to search for hybrid mesons with exotic quantum numbers using a beam of linearly polarized photons incident on a liquid hydrogen target. The linearly polarized photon beam in Hall D will be produced via the coherent bremsstrahlung process by $12 \mathrm{GeV}$ electrons in a thin diamond radiator. Coherent bremsstrahlung has been successfully used to produce linearly polarized photon beams at various experimental facilities $[2,3]$. Coherent radiation from a diamond crystal lattice results in sharp monochromatic peaks in the photon energy spectrum. The peak energy depends on the relative orientation of the electron beam direction and the crystal lattice, and can be

\footnotetext{
Notice: Authored by Jefferson Science Associates, LLC under U.S. DOE Contract No. DE-AC0506OR23177. The U.S. Government retains a non-exclusive, paid-up, irrevocable, world-wide license to publish or reproduce this manuscript for U.S. Government purposes.

*Corresponding author. Tel.: +1 757269 5553; fax: +1 7572696331 .

Email address: somov@jlab.org (A.Somov)
} 
adjusted in the experiment by rotating the diamond. The GlueX photon energy region of interest corresponding to the main coherent peak is between $8.4 \mathrm{GeV}$ and $9.1 \mathrm{GeV}$. The fraction of linearly polarized photons can be increased by passing a photon beam through a $3.4 \mathrm{~mm}$ diameter collimator situated about $75 \mathrm{~m}$ downstream of the radiator. The collimator preferentially filters out photons produced via coherent bremsstrahlung, which have a substantially larger mean emission angle. The collimated photons propagate toward the GlueX target. The energy of a beam photon can be inferred by analyzing the momentum of the electron after it has radiated the photon, given that the primary beam energy is known. This so-called "tagged" electron is deflected in a $6 \mathrm{~m}$ long dipole magnet operated at a field of $1.8 \mathrm{~T}$ and is subsequently registered in the tagging microscope or broad-band hodoscope scintillator counters.

One of the key components of the Hall D photon beam line is the magnetic pair spectrometer, which is installed after the photon collimator in front of the GlueX detector. The spectrometer will reconstruct the energy of a beam photon by detecting the $e^{ \pm}$pair produced by the photon in a thin converter. The main purpose of the spectrometer is to measure the spectrum of the collimated photon beam and determine the fraction of linearly polarized photons in the coherent peak energy region. It will also monitor the photon beam flux and can be used for the energy calibration of the tagging hodoscope and microscope detectors. The description of the Hall D pair spectrometer will be presented in Section 2. The design of the pair spectrometer high-granularity hodoscope and light collection measurements from hodoscope scintillator tiles will be described in Section 3 and Section 4, respectively.

\section{Pair Spectrometer}

Layout of the Hall D pair spectrometer is presented in Fig. 1. Electron-positron pairs are created by beam photons inside a thin converter with a typical thickness ranging between $10^{-4}-10^{-2}$ radiation lengths. The choice of the converter thickness depends on the photon beam flux. The maximum flux for GlueX physics runs is expected to be $5 \cdot 10^{7} \gamma / \mathrm{sec}$ in the coherent peak energy region. Three converters with different thicknesses are installed in a movable fork, that can insert one of them into the photon 


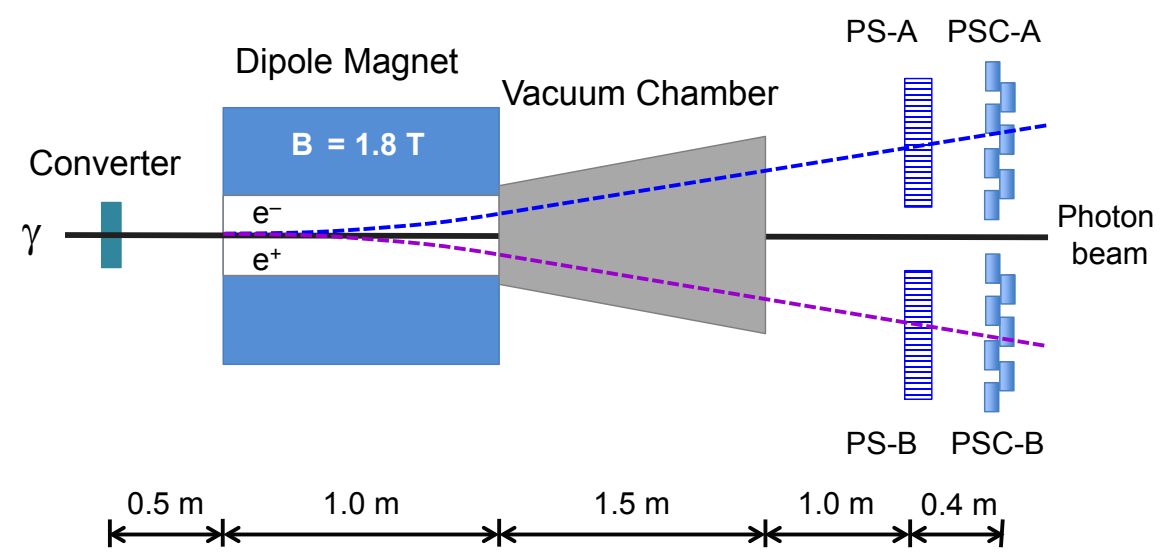

Figure 1: Schematic top view of the Hall D pair spectrometer. PS and PSC denote the hodoscope and coarse scintillator counters, respectively.

beam. Produced leptons are deflected in a 18D36 dipole magnet with an effective field length of about $0.94 \mathrm{~m}$. The magnet was brought from Brookhaven National Laboratory and was modified at Jefferson Lab by reducing the pole gap from 6 inches to 3 inches. The magnet is operated at a nominal field of $1.8 \mathrm{~T}$. A 1.5 meter long vacuum chamber is installed after the magnet. Electrons and positrons are registered in two layers of scintillator detectors: a high-granularity hodoscope and a set of coarse counters, denoted in Fig. 1 as PS and PSC, respectively. The detectors are organized into two arms positioned symmetrically with respect to the photon beam line. Each detector arm covers a momentum range of $e^{ \pm}$between $3.0 \mathrm{GeV} / \mathrm{c}$ to $6.2 \mathrm{GeV} / \mathrm{c}$, corresponding to reconstructed photon energies between $6 \mathrm{GeV}$ and $12.4 \mathrm{GeV}$. Relatively large acceptance of the hodoscope allows one to reconstruct photons with energies in the coherent peak energy region and also in the range near the beam end-point energy of $12 \mathrm{GeV}$. This can be used for the energy calibration of the hodoscope detectors.

The high-resolution hodoscope is used for precise measurements of $e^{ \pm}$momenta. The hodoscope consists of a set of thin scintillator tiles, stacked together. Momentum of the reconstructed lepton is related to the $x$-coordinate of each tile, where $x$ is an axis perpendicular to the beam line. Hodoscope tiles will be calibrated in units of lepton 
energy, the energy scale will be used hereafter in the text. The hodoscope momentum resolution was studied using a detailed Geant detector simulation. The hodoscope energy resolution varies between $12 \mathrm{MeV}$ and $20 \mathrm{MeV}$ for $6 \mathrm{GeV}$ and $12 \mathrm{GeV}$ reconstructed photons, respectively. Light from each scintillator tile is detected using a Hamamatsu silicon photomultiplier (SiPM). The detailed description of the hodoscope will be presented in Section 3 .

Sixteen coarse scintillator counters, eight in each detector arm, are positioned about $40 \mathrm{~cm}$ behind the hodoscope. The counters are $4.4 \mathrm{~cm}$ wide in the direction perpendicular to the lepton trajectory, $2 \mathrm{~cm}$ thick, and $6 \mathrm{~cm}$ in height. Hamamatsu R6427-01 PMTs are used to detect the scintillation light. The counters are used to produce a pair spectrometer trigger by requiring a coincidence of hits in the two detector arms. They also help to reduce background originating from interactions of $e^{ \pm}$inside the magnet pole edges to the level below $1 \%$ by constraining $e^{ \pm}$trajectories. Counter rates depend on the converter thickness and photon beam flux. The maximum rate will not exceed $10 \mathrm{kHz}$ per PSC counter during GlueX operation.

Signals from both the hodoscope and coarse counters are digitized using a twelvebit multi-channel flash ADC operated at a sampling rate of $250 \mathrm{MHz}$ [5]. The PSC counters are also instrumented with TDCs, with a channel width of 60 ps [6]. The time resolution is expected to be better than 200 ps. This resolution will allow one to distinguish the electron beam bunch where the bremsstrahlung photon is emitted and therefore relate hits from the pair spectrometer and tagging detectors originating from the same event.

\section{Pair Spectrometer Hodoscope}

\subsection{Design and Fabrication}

The pair spectrometer hodoscope consists of 145 rectangular tiles made of EJ-212 scintillator [4], stacked together as shown in Fig. 2. The tile height is $3 \mathrm{~cm}$ and the length along the electron path in scintillator is $1 \mathrm{~cm}$. Tiles with two different widths, in the direction perpendicular to the electron trajectory, of $2 \mathrm{~mm}$ and $1 \mathrm{~mm}$ are used. Forty $1 \mathrm{~mm}$ wide tiles are instrumented in the detector high-energy region around 6 
$\mathrm{GeV}$ in order to provide better energy resolution in this energy range. The energy bin size of the hodoscope tiles varies depending on the lepton energy and constitutes about $13 \mathrm{MeV}$ and $24 \mathrm{MeV}$ for $3 \mathrm{GeV}$ and $6 \mathrm{GeV}$ leptons, respectively. Tiles are optically isolated using $10 \mu \mathrm{m}$ aluminized Mylar foil. This reflective foil also covers the bottom of the tile assembly. The deflection angle of leptons in the dipole magnet depends on the lepton momentum; the angular spread is about 5 degrees between $3 \mathrm{GeV}$ and 6.2 $\mathrm{GeV}$ electrons. To account for the angular dependence, hodoscope tiles are organized into 18 groups. Each group is tilted by about 0.3 degrees. This was done by attaching a shim, $\mathrm{a} \sim 50 \mu \mathrm{m}$ thick adhesive strip, to one side of the aluminum foil after each group.

Light from a tile is collected using two $20 \mathrm{~cm}$ long $1 \mathrm{~mm}$ x $1 \mathrm{~mm}$ square doubleclad BCF-92 wave-length shifting (WLS) fibers, each of which is glued to the side of the tile using BC 600 Optical Cement. A tile assembly with two WLS optical fibers is shown in Fig. 3. The peak of the emission spectrum for EJ-212 scintillator occurs at the wavelength of $423 \mathrm{~nm}$, which couples well with the absorption spectrum of the BCF-92 fiber. Light is subsequently reemitted inside the fiber in the green range with an emission peak of $492 \mathrm{~nm}$.

Collected light is transmitted to the end of the WLS fiber. A pair of fibers from each tile is inserted into a hole in an aluminum mounting plate, as shown on the upper plot of Fig. 2. An electronics circuit board containing 145 photo detectors is attached to the mounting plate. Each photo sensor is coupled to two WLS fibers from a single tile. The light detection is performed using Hamamatsu surface mount S10931-050P silicon photomultipliers with an effective photosensitive area of $3 \mathrm{~mm}$ x $3 \mathrm{~mm}$ and a pixel size of $50 \mu \mathrm{m}$. These sensors have a photon detection efficiency (PDE) larger than $20 \%$ at a wavelength of $500 \mathrm{~nm}$ and a typical gain of about $7 \cdot 10^{5}$ [7]. The electronics board with SiPMs is presented in Fig. 4. The SiPMs are arranged in two arrays of 3 x 35 and $5 \times 8$ sensors, which are connected to $2 \mathrm{~mm}$ and $1 \mathrm{~mm}$ tiles, respectively. SiPMs are optically isolated using a plastic spacer.

\subsection{Electronics}

The hodoscope front end electronics consists of an amplifier and a SiPM bias voltage control circuit developed at Jefferson Lab. Signals from the SiPMs are amplified 
using the amplifier with a gain of about a factor of 20. The amplifier is based on commercially available devices (operational amplifiers) with $3 \mathrm{GHz}$ bandwidth. Pulse shaping is employed to compensate for the characteristically high SiPM capacitance and package inductance. The impulse response shows rise and fall times of $3 \mathrm{~ns}$ and with trans-impedance gain of $1 \mathrm{mV} / \mu \mathrm{A}$. The SiPM operating bias voltage is about 73 V. The nominal bias setting is as specified by Hamamatsu (1 V over voltage) and fed to the SiPM through a resistive network employing a thermistor and a linearizing resistor. The hodoscope control electronics supply individually adjusted voltages to groups of $5 \mathrm{SiPM}$ channels; inside the group the voltage is adjusted among channels using resistors. The thermistor senses the average temperature of closely packed SiPMs in thermal equilibrium via a heat spreader PCB layout, thus forming a well controlled loop. The commercially available bias power supply has very low noise characteristics and is well regulated to less than $1 \mathrm{mV}$ long term. The supply allows the user to monitor and adjust the levels as needed and if required. The optimal bias setting will be determined based on experimental conditions. Amplified SiPM signals are digitized by flash ADCs positioned in two readout VXS crates. An example of the flash ADC signal pulse obtained from a scintillator tile is shown in Fig. 5. The ADC sampling time is $4 \mathrm{~ns}$.

After fabrication, the performance of SiPMs and electronics were tested using a picoseconds laser light pulser ${ }^{1}$. The typical rise time of a SiPM pulse produced by a laser pulser is about $6 \mathrm{~ns}$ and the corresponding fall time is $45 \mathrm{~ns}$. The pulser trigger output was used to initiate the flash ADC readout. SiPM amplitudes digitized by ADC are integrated in a time window of 60 ns. A typical ADC spectrum is shown in Fig. 6. Peaks on this plot correspond to the number of pixels fired in the SiPM. The position of a single-pixel peak depends on the combined gain of a SiPM and electronics. The single-pixel peak distribution measured for all hodoscope SiPMs is presented in Fig. 7. The solid curve corresponds to the fit of this distribution to a Gaussian function. The relative gain variation is found to be smaller than $1.5 \%$.

\footnotetext{
${ }^{1}$ Hamamatsu PLP-10 light pulser was used to test the hodoscope electronics.
} 


\section{Light Collection Studies}

\subsection{Beam Test}

During the design phase of the hodoscope, we studied light collection from scintillator tiles using a detector prototype that consisted of fourteen $2 \mathrm{~cm} \mathrm{x} 1 \mathrm{~cm} \times 1 \mathrm{~mm}$ and five $2 \mathrm{~cm} \times 1 \mathrm{~cm} \times 2 \mathrm{~mm}$ tiles. Tiles were made from the same scintillator type EJ-212 but had smaller height of $2 \mathrm{~cm}$. Light collection was performed using a single BCF-92 fiber, which was glued to one side of the tile using DYMAX UV optical adhesive. Light was detected by Hamamatsu SiPMs S10362-11-025C and S10362-11050C, which have the pixel size of $25 \mu \mathrm{m}$ and $50 \mu \mathrm{m}$, respectively. These SiPMs have a smaller sensitive area of $1 \mathrm{~mm} \times 1 \mathrm{~mm}$. Prototypes of the SiPM amplifier and the bias control circuit were used. The trigger was provided by a small plastic scintillator mounted behind the tiles. The trigger scintillator was connected to a Philips XP 1911 photomultiplier tube via two optical fibers. The setup used in the beam test is shown in Fig. 8. SiPM pulses were digitized using a flash ADC in a VXS crate.

The hodoscope prototype was positioned in the tagger area of the experimental Hall B at Jefferson Lab [3] and was operated concurrently with the CLAS detector. The CLAS used a secondary beam of photons produced in the tagger area by the Jefferson Lab electron beam in a thin radiator via the bremsstrahlung process. A beam electron that radiates a photon is deflected in the dipole tagger magnet and sent to the floor of the experimental hall. The prototype was placed inside a light-tight box and positioned on the floor of the tagger area. The hodoscope was oriented in such a way that it faced normal to the path of electrons, i.e., electrons travel $1 \mathrm{~cm}$ through the tile. A schematic view of the tagger area and the location of the prototype is shown in Fig. 9. Dashed curves on this plot denote trajectories of tagged electrons with the fractional energy $k / E_{0}$, where $k$ is the tagged electron energy and $E_{0}$ is the electron beam energy. Data was taken with an electron beam energy of $2.54 \mathrm{GeV}$, corresponding to a tagged electron energy of about $1.4 \mathrm{GeV}$.

The amount of light collected from a tile was measured in units of pixels fired in the SiPM. The average number of pixels was estimated to be

$$
N_{\text {pixels }}=A_{\text {peak }} / A_{\text {single }},
$$


where $A_{\text {peak }}$ is the ADC value corresponding to the maximum of an ADC spectrum measured in the beam test and $A_{\text {single }}$ is the ADC value of a single pixel peak. The position of the single pixel peak was calibrated using a LED pulser. Exact counting of the number of photons that induce pixel-breakdowns is complicated due to the optical cross-talk and after-pulses in the SiPM. The cross-talk is produced by secondary photons emitted in the pixel breakdown that trigger an additional avalanche in a neighboring pixel. The after-pulsing is believed to be produced by charge carriers that are released from the substrate after some delay from the avalanche resulting in consecutive SiPM pulses. The cross-talk and after-pulse probabilities for the SiPMs used in the hodoscope are expected to be relatively small, less than $10 \%$ [8]. For the prototype operational bias voltage (about $1 \mathrm{~V}$ over) we expect on average about 0.2 pixels fire per incident photon. SiPM operational voltages for the final detector will be adjusted according to the experimental conditions and detector performance.

The beam test results are listed in Table 1. The amount of light collected from the $1 \mathrm{~mm}$ and $2 \mathrm{~mm}$ wide tiles is found to be almost the same and corresponds to about 29 pixels fired in the SiPM with the pixel size of $50 \mu \mathrm{m}$. This indicates that the light collection mainly depends on the surface of the WLS fiber glued to the tile, which is similar for $1 \mathrm{~mm}$ and $2 \mathrm{~mm}$ tiles. The fewer number of fired pixels measured with a $25 \mu \mathrm{m} \mathrm{SiPM}$ is consistent with about a factor of two smaller photon detection efficiency of these SiPMs compared with $50 \mu \mathrm{m}$ SiPMs. The smaller pixel fill factor (the ratio of the active area of a pixel to the entire area) accounts for the smaller PDE for the $25 \mu \mathrm{m}$ S10362-11-025C SiPMs. We estimate that errors on light collection measurements are dominated by alignment of the WLS fiber with respect to the SiPM. The sensitive area of SiPMs used in the prototype is the same as the fiber cross section.

Light collection from the final hodoscope detector will be performed using two WLS fibers and SiPMs with a factor of 9 larger effective area. Therefore, we expect about a factor of two more light collected from the tile and a SiPM response corresponding to about 60 fired pixels. This amount of light should be sufficient to provide a high detection efficiency for $e^{ \pm}$pairs in the hodoscope. The hodoscope can be operated at relatively small ADC thresholds because a coincidence of hits in two detector arms 


\begin{tabular}{l|c|c|c}
\hline Tile width $(\mathrm{mm})$ & \multicolumn{2}{|c|}{1} & 2 \\
\hline \hline SiPM pixel size $(\mu \mathrm{m})$ & 25 & 50 & 50 \\
\hline Light detected (pixels) & $16.1 \pm 2.1$ & $28.7 \pm 3.4$ & $29.2 \pm 3.6$ \\
\hline
\end{tabular}

Table 1: The amount of light in units of fired pixels seen by SiPM for various tiles used in the beam test.

is required.

\subsection{Cosmic Setup}

During the fabrication of scintillator tiles for the final hodoscope, we checked light collection using a cosmic ray setup presented in Fig. 10. Muon candidates were selected using two small trigger scintillator counters with an effective area of $6 \mathrm{~mm} x$ $3 \mathrm{~cm}$ and thickness of $2 \mathrm{~mm}$. Counters were positioned perpendicular to each other and formed an overlap region of $6 \mathrm{~mm} \times 6 \mathrm{~mm}$. The distance between scintillators was about $20 \mathrm{~cm}$. A hodoscope tile was placed between these counters, in such a way that cosmic particles go perpendicular to the tile, in the middle. The average muon path length in the scintillator corresponds to the tile width. A lead brick about $10 \mathrm{~cm}$ thick was position above the bottom scintillator to filter low-energy particles. Light detection was performed using a Hamamatsu S10931-050P SiPM instrumented with a single-channel amplifier. The amplifier gain was about a factor of ten smaller than that used in the hodoscope. A typical ADC spectrum obtained from the SiPM is shown on the top plot of Fig. 11. The average amount of light collected from $2 \mathrm{~mm}$ wide tiles corresponds to about 18 pixels fired in the SiPM. A Sr90 source was used to check quality of some selected fabricated tile assemblies. The SiPM spectrum obtained from a $2 \mathrm{~mm}$ wide tile using the radioactive source is shown on the bottom plot of Fig. 11 for comparison.

\section{Summary}

We have described the design and fabrication details of the pair spectrometer hodoscope, an array of thin scintillator tiles. Light from each tile is detected using a 3 
mm x $3 \mathrm{~mm}$ Hamamatsu SiPM. A detector prototype was built to perform light collection studies using relativistic electrons produced in the experimental Hall B at Jefferson Lab. The amount of light for $1 \mathrm{~mm}$ and $2 \mathrm{~mm}$ tiles measured with the prototype corresponds to a SiPM response of about 29 fired pixels. At least a factor of two more light is expected for the final hodoscope for which the light collection is performed using two WLS fibers and tiles with larger size, i.e., larger light collection surface, are used. This amount of light is sufficient to provide a high detection efficiency of leptons by the hodoscope. Two arms of the hodoscope detector were commissioned and installed in the experimental Hall D.

\section{Acknowledgments}

This work was supported by the Department of Energy. Jefferson Science Associates, LLC operated Thomas Jefferson National Accelerator Facility for the United States Department of Energy under contract DE-AC05-06OR23177. We would like to thank the CLAS Collaboration for an opportunity to conduct the beam test at Hall B and especially Eugene Pasyuk and Sergey Boyarinov for the assistance with the beam test. We are also thankful to Slava Razmyslovich for his help with preparing some technical drawings for the hodoscope.

\section{References}

[1] JLab Experiment E12-06-102, (2006) http://www.jlab.org/exp_prog/ proposals/06/PR12-06-102.pdf.

[2] G. Diambrini-Palazz et al. Phys. Rev. Lett. 25, 478 (1970); R. O. Avakyan et al., Proc. High En. Phys. Conf. , Dubna 2 (1971); R. Schwitters, SLAC-TN-70-032; W. Kaune et al., Phys. Rev. D 11, 478 (1975); A. Jackson, Nucl. Instrum. Meth. 129, 73 (1975); R. Brockmann et al., BONN-IR-79-25 (1979); F. Rambo et al., Phys. Rev. C 58, 489 (1998); D. Lohmann et al., Nucl. Instrum. Meth. A 343, 494 (1994).

[3] D. I. Sober et al., Nucl.Inst.and Meth. A, 440 (2000),p.263. 
[4] ELJEN Technology Plastic Scintillators http://www.eljentechnology .com.

[5] F. Barbosa et al., Proceedings of IEEE Nuclear Science Symposium, Hawaii, USA (2007).

[6] F. Barbosa et al., Proceedings of IEEE Nuclear Science Symposium, Virginia, USA (2002).

[7] Hamamatsu Corporation, MPPC S10931-050P, http://www . electronicsdatasheets.com/pdf-datasheets/hamamatsu/s10931050p.

[8] P. Eckert, H. C. Schultz-Coulon, W. Shen, R. Stamen and A. Tadday, Nucl. Instrum. Meth. A 620, 217 (2010). 

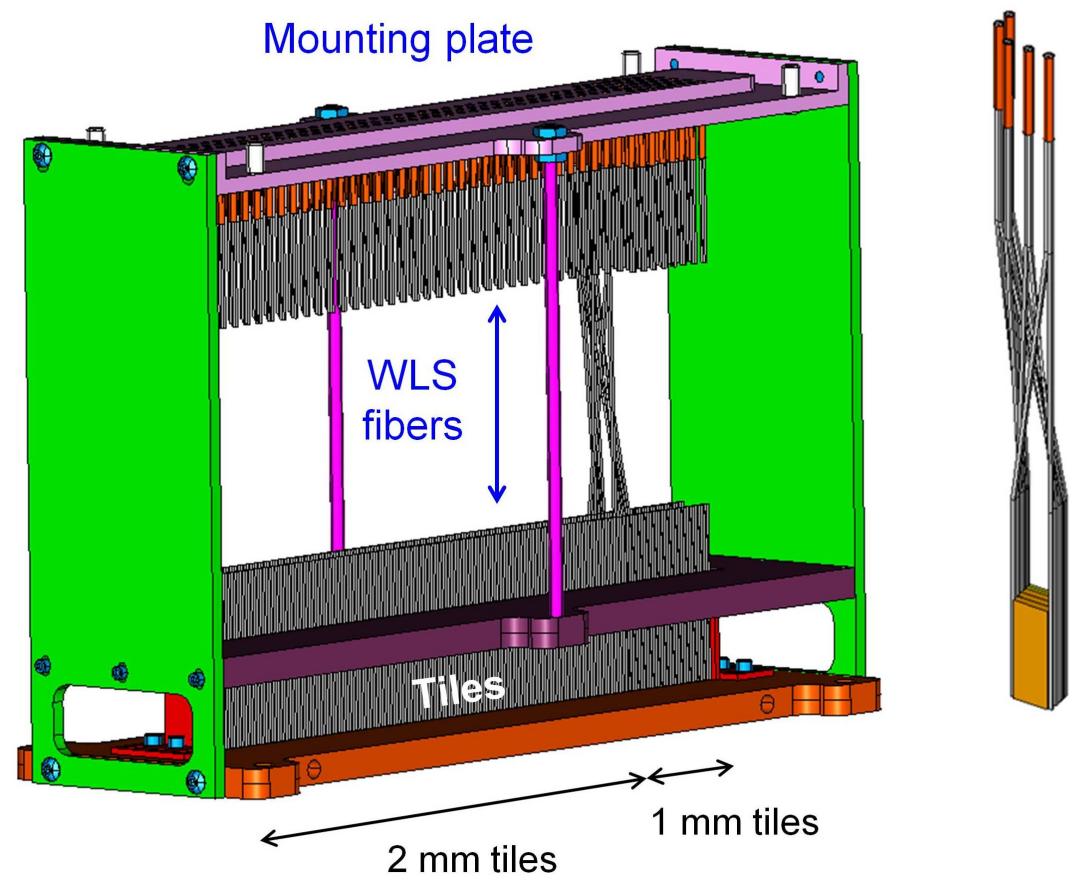

WLS

fibers

Tiles

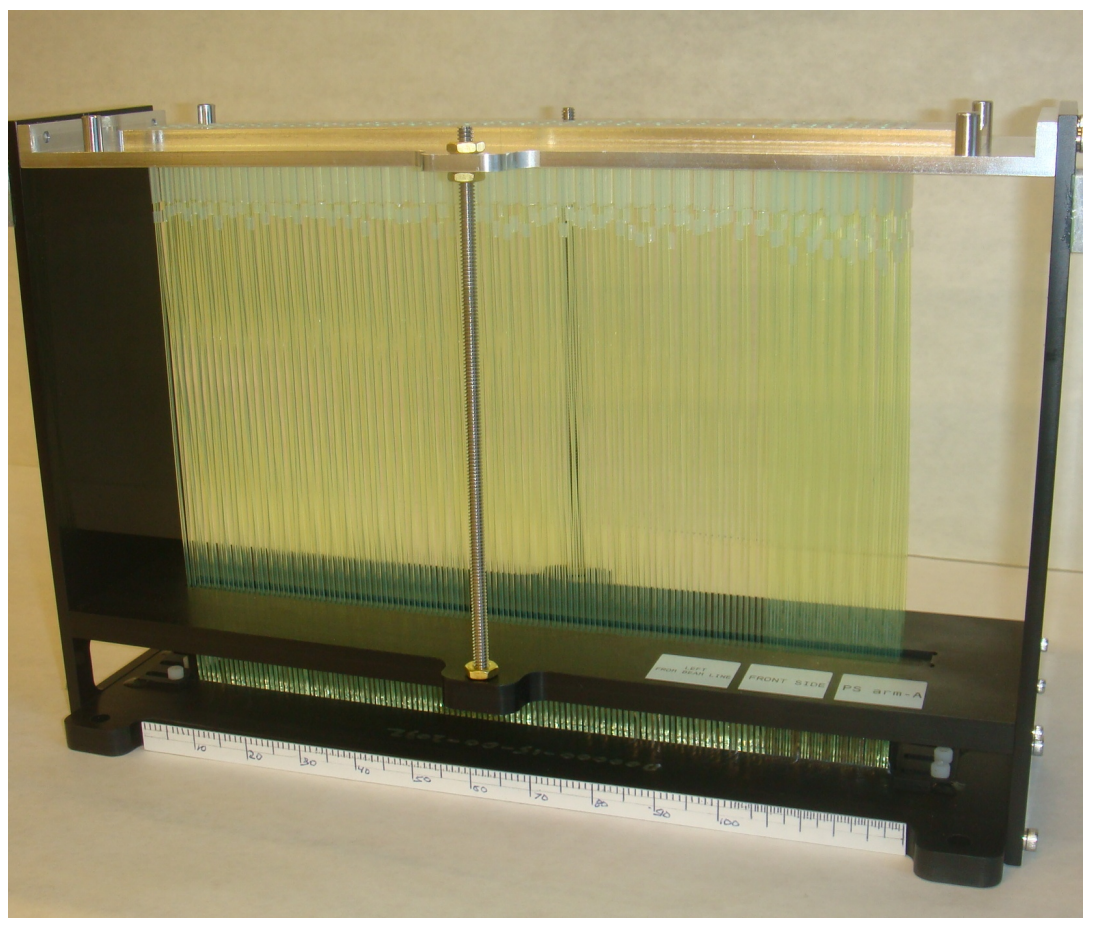

Figure 2: Schematic view of the pair spectrometer hod 2 scope (top). Light from scintillator tiles is collected using WLS fibers, which are inserted into holes in the mounting plate. Fabricated detector is shown in the bottom picture. 


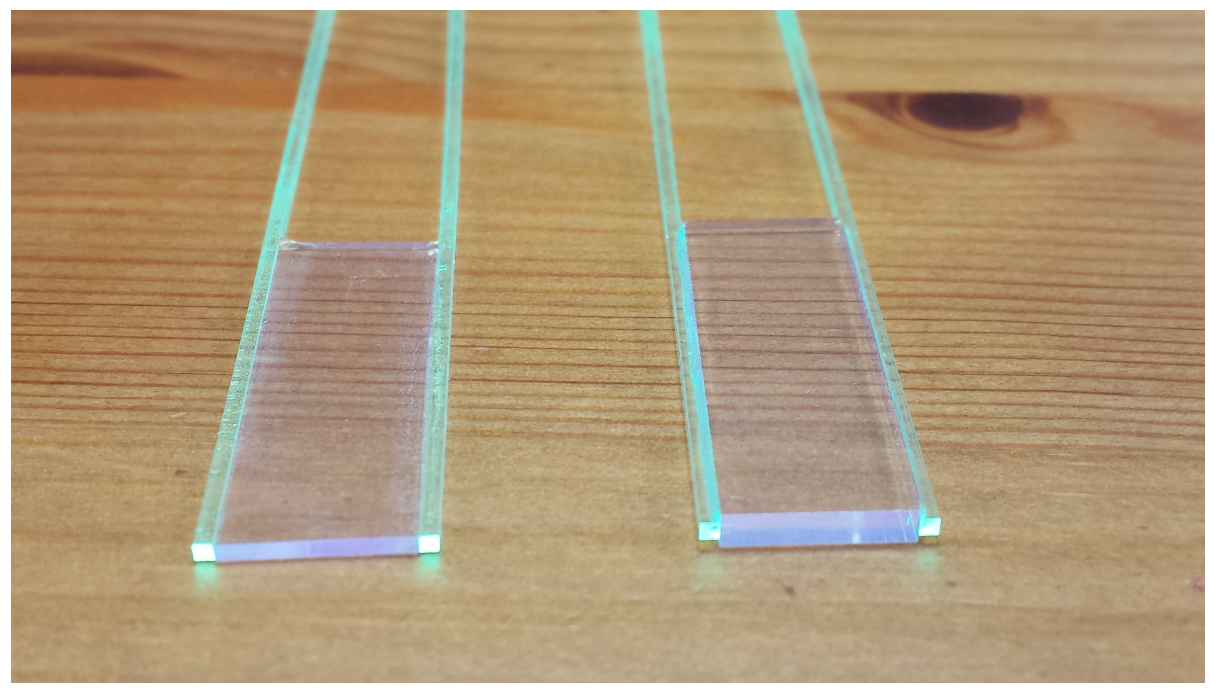

Figure 3: Scintillator tiles with two WLS fibers glued to sides of each tile: $3 \mathrm{~cm} \times 1 \mathrm{~cm} \times 1 \mathrm{~mm}$ tile (left) and $3 \mathrm{~cm} \times 1 \mathrm{~cm} \times 2 \mathrm{~mm}$ tile (right).

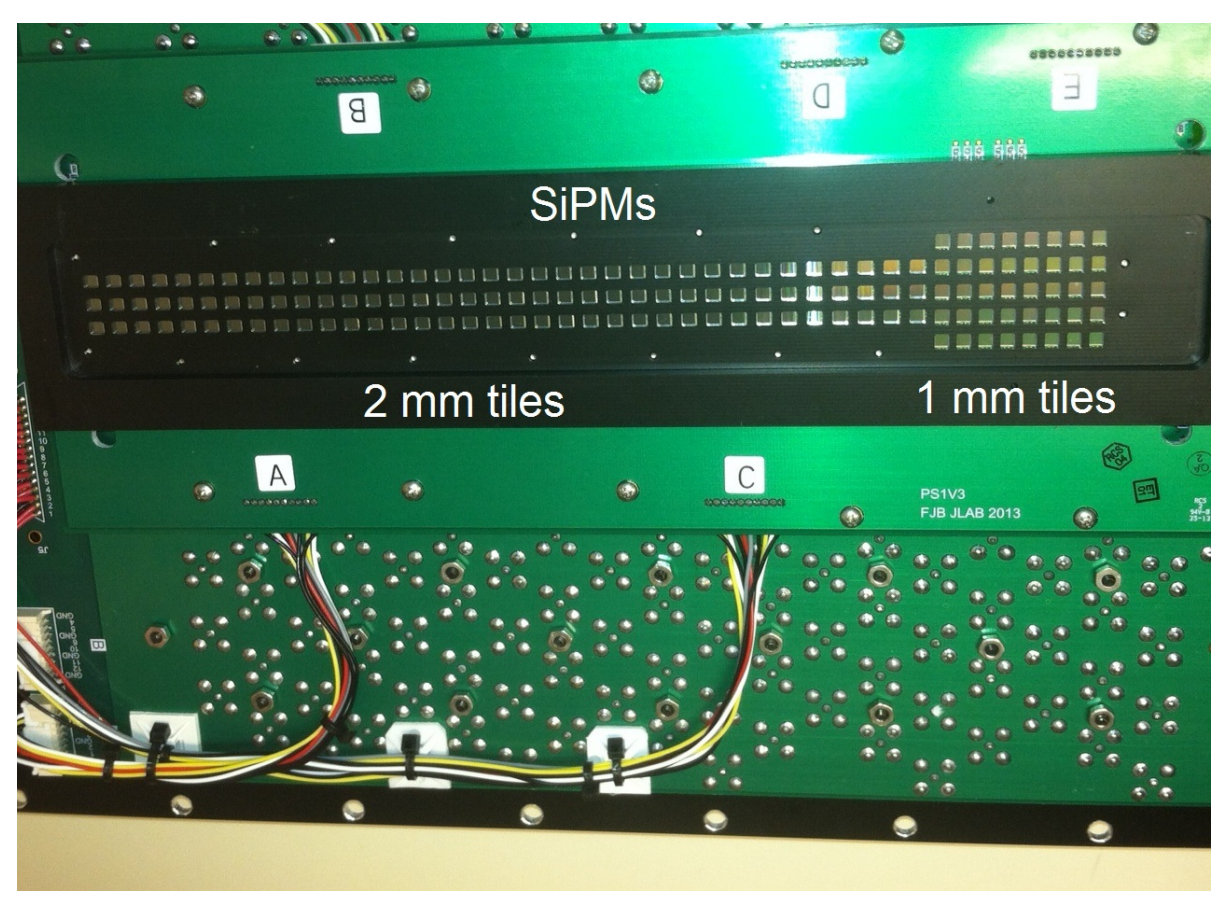

Figure 4: Electronics board with 145 silicon photomultipliers. 


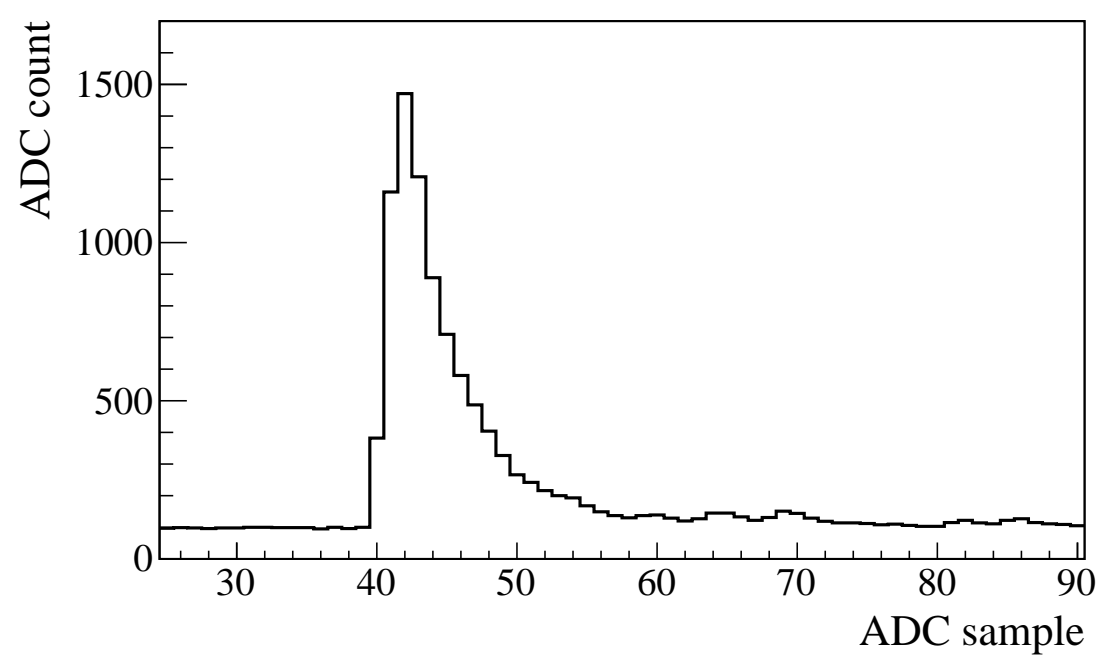

Figure 5: A typical flash ADC signal pulse obtained from a scintillator tile. 


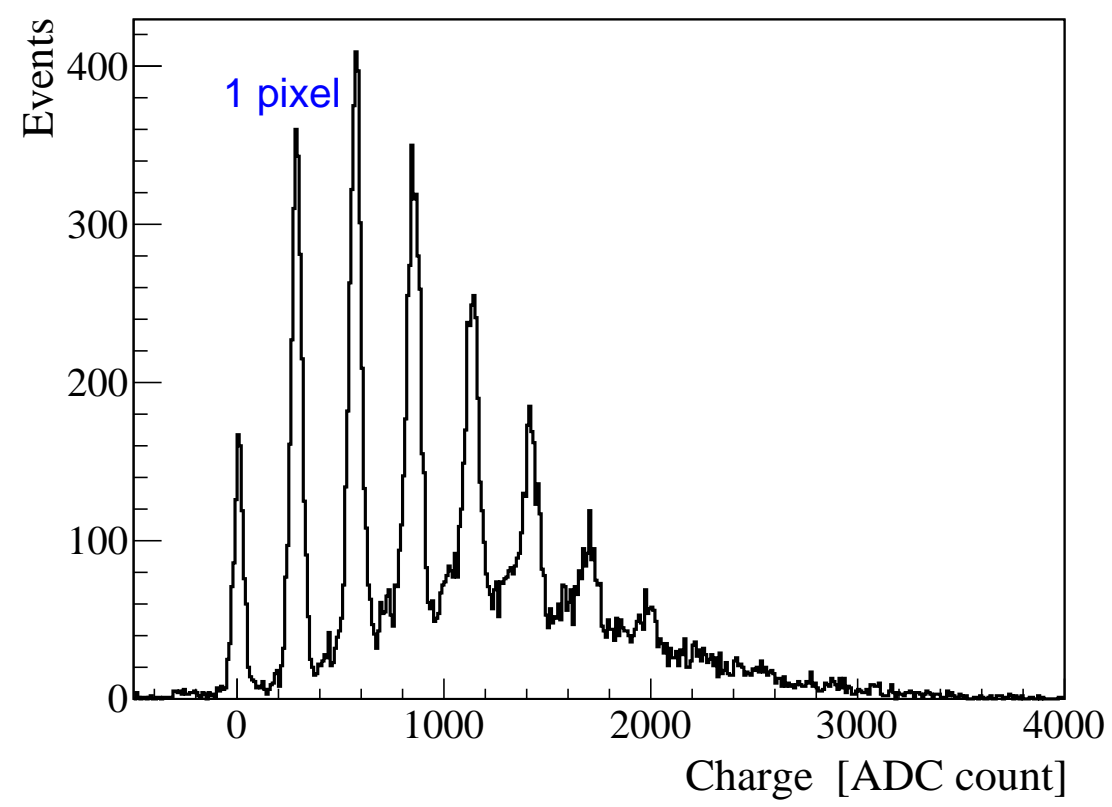

Figure 6: An example of the ADC spectrum obtained from SiPMs during electronics tests using a laser pulser. The first peak on the spectrum around ADC count zero corresponds to the ADC pedestal. 


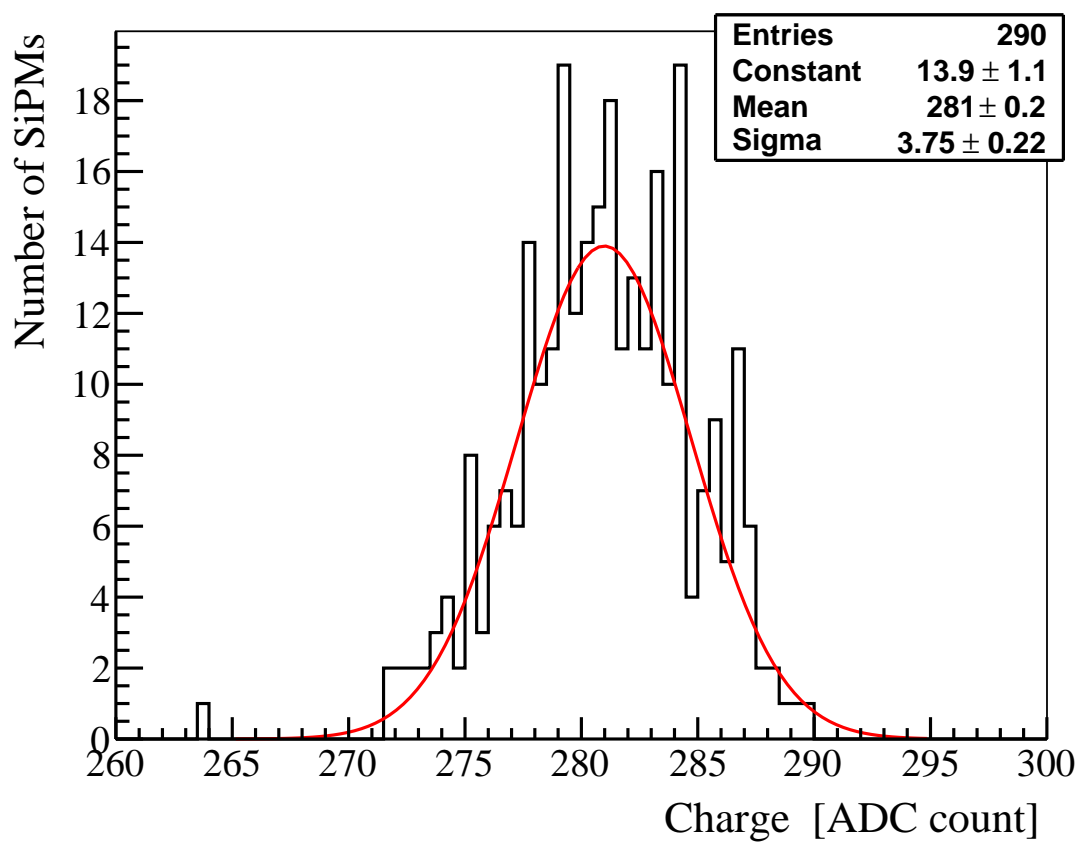

Figure 7: Distribution of the single-pixel peak measured by flash ADC for 290 hodoscope channels.

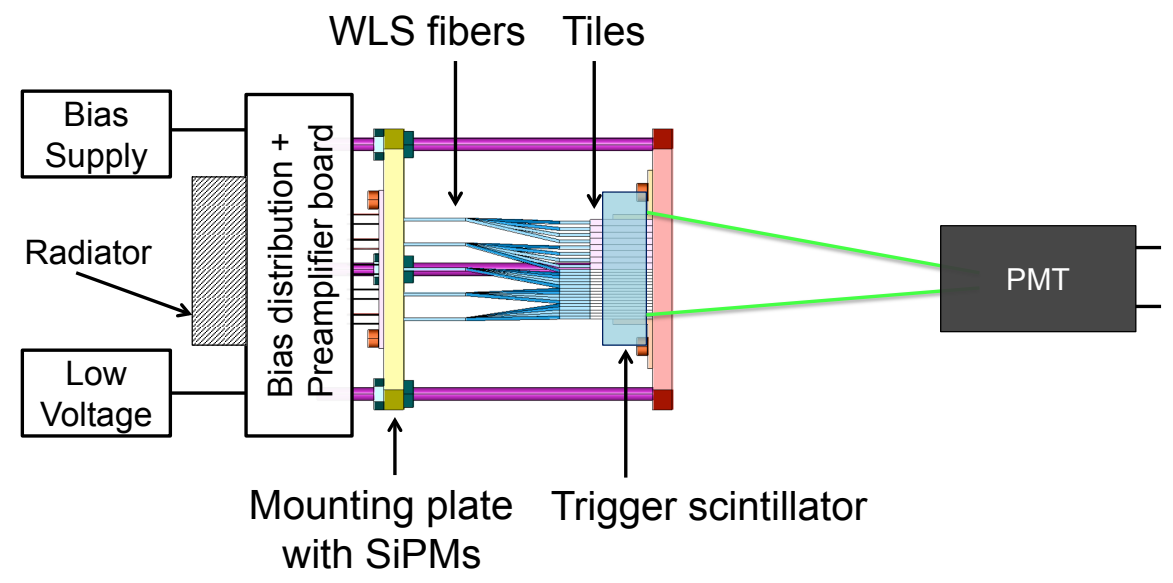

Figure 8: Top view of the hodoscope prototype with the trigger scintillator used in the beam test in experimental Hall B. 


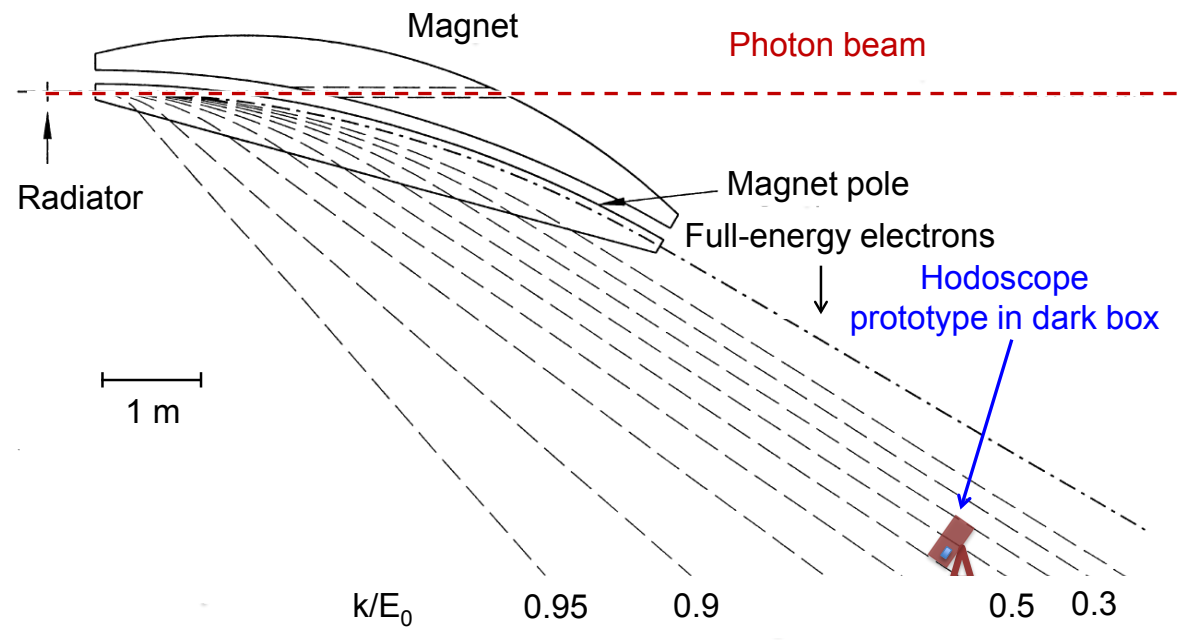

Figure 9: Hall B tagger system. Location of the hodoscope prototype under the Hall B tagger. Dashed curves denote trajectories of tagged electrons.

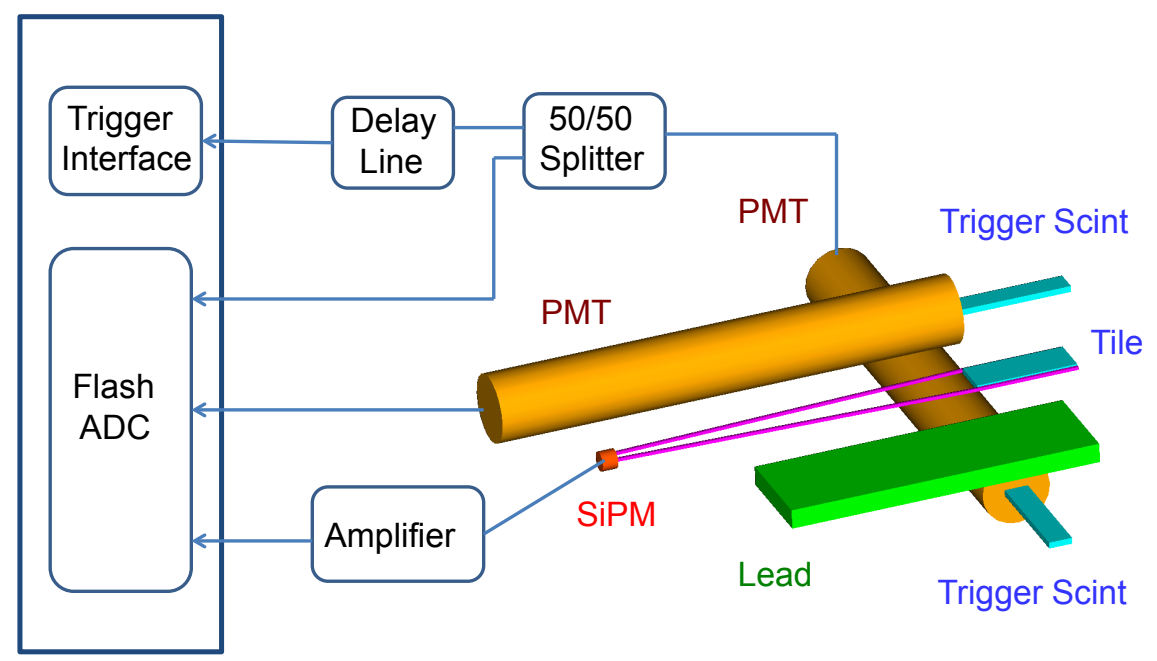

Figure 10: A cosmic ray setup. 

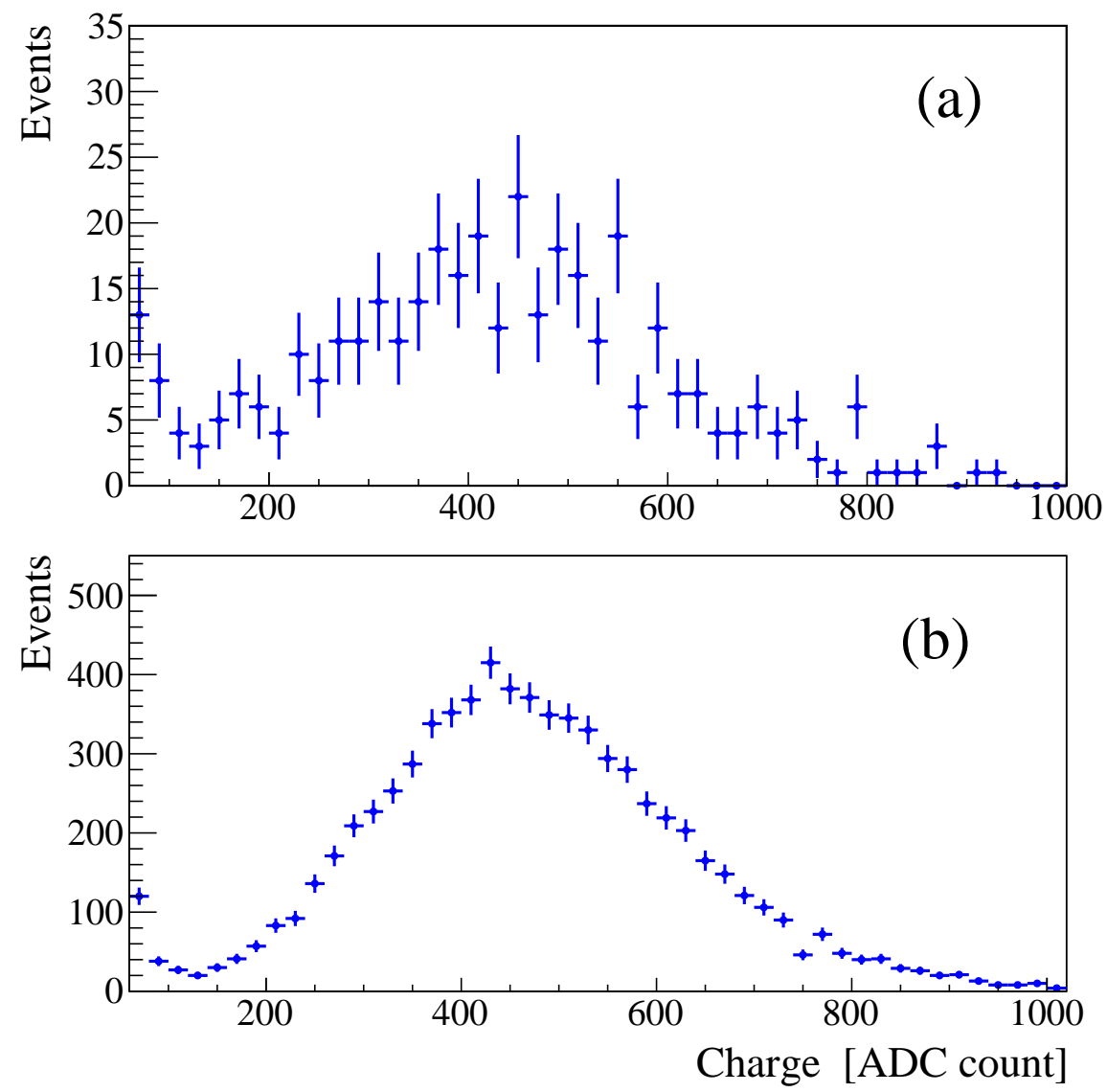

Figure 11: ADC spectra for a $2 \mathrm{~mm}$ wide tile measured with cosmic muons (a) and a ${ }^{90} \mathrm{~S} r$ radioactive source (b) 